\title{
Functional Hamstring to Quadriceps Strength Ratio (H:Q) and Hamstrings Injury of Soccer Players: A Qualitative Analysis
}

\author{
Muhammad Shahidul Islam ${ }^{1}$ and Anup De ${ }^{2 *}$ \\ ${ }^{1}$ Assistant Director, Office of Physical Education, Shahjalal University of Science and Technology, Bangladesh \\ ${ }^{1}$ Research Scholar, Department of Physical Education, Visva-Bharati University, West Bengal, India \\ ${ }^{2}$ Research Scholar, UGC-Senior Research Fellow, Exercise and Sports Physiology Laboratory, Department of Physical Education, Visva- \\ Bharati University, West Bengal, India
}

Received: 眥 October 25, 2018; Published: 些 October 31, 2018

*Corresponding author: Anup De, Research Scholar, UGC-Senior Research Fellow, Exercise and Sports Physiology Laboratory, Department of Physical Education, Visva-Bharati University, West Bengal, India

\begin{abstract}
Background: Soccer players are required to perform explosive and powerful movements as the game is very competitive and intermittent nature. Functional hamstring to quadriceps strength ratio can plays a significant role to perform these movements. The imbalance of this ratio occurs hamstring muscles injury. Current researchers found the clear connection between functional hamstring to quadriceps strength ratio and the non-contact hamstrings injury of soccer players.
\end{abstract}

Objective: The aim of the review study was to analyze the functional strength ratio between hamstrings and quadriceps as a measure of hamstring injury.

Method: Researchers collected scientific evidences through electronic online databases; PubMed, Embase, Medline, Google scholar, Web of science, PsycInfo, and critically analyzed the entire relevant article according to the nature of this study.

Findings: Nordic hamstring strengthening exercise and FIFA 11+ injury prevention programme may reduce the rate and severity of hamstring muscle injuries and also develop the eccentric strength of hamstrings especially on biceps femoris long head muscle fascicles.

Conclusion: Hamstring to quadriceps strength ratio need to be maintaining regularly to overcome hamstrings injury. Progressive and systematic nordic hamstring strengthening exercise can decrease hamstring lesions and reduction of re-injury.

Keywords: Hamstring to quadriceps strength ratio; Biceps femoris long head; Nordic hamstring exercise; Eccentric strength; Muscles injury; Soccer

\section{Introduction}

Soccer player's continuously suffering from several lower extremity injuries. Hamstrings muscle injury is one of them. The game of soccer is body contact and intermittent nature. Most of the hamstring's injury occurred due to less stretchable and strengthening ability of the muscles. Therefore, this injury appeared by non-body contact situation even though game has body contact difficulty. Muscle strength is one of the key factors in high performance and is an important indicator of the effectiveness of injury prevention in athletes [1,2]. However, one of the most used methods to assess muscle strength balance between dominant/ non-dominant and antagonist/agonist is isokinetic testing [2,3]. Therefore the sports scientist wants to develop a number of muscle strength methods especially lower limb strength.

The hamstrings are a collection of muscles and their tendons at the back of the upper limb. The muscles bend the knee joint, adduct the leg, and extend the thigh to the rear end of the body. Strength of the hamstrings muscle alone is not the only main factor in injury prevention. The strength ratio of hamstring to quadriceps $(\mathrm{H}: \mathrm{Q})$ is necessary to provide sufficient development process for playing without injury. Quadriceps is naturally robust than the hamstring. So that, sometime this can overpower their hamstrings. This imbalance of hamstring to quadriceps strength ratio is often the reason of hamstrings injury [4,5]. Hamstring to quadriceps the highest torque strength ratio (H:Q) is one of the most usually assessed. To examine the functional capacity, the ratio of strength (H:Q) of agonist to antagonist knee muscles has been used [1]. 
The collective term 'hamstrings' refers to four muscles located in the posterior compartment of the thigh semimembranosus (SM), semitendinosus (ST), biceps femoris long head (BFlh) and biceps femoris short head (BFsh). These muscles are multiarticular and susceptible to strain injury, which is often recurrent [6-8]. Quadriceps and hamstrings strength are needed for physical performance. These two muscles are functional antagonists; contraction of the quadriceps results in knee extension, while contraction of the hamstrings results in a flexion of the knee joint [9]. As the intermittent nature of the soccer match, the player would be tired when the match goes towards ending. So, the most common hamstrings injury occurred due to tiredness and fewer stretchable ability of the respective muscle $[10,11]$. However, thigh muscles indirect injuries are one of the most common injured areas in soccer [12]. Additionally, the most usually injured part in sport are the hamstrings, rectus femoris and medial head of the gastrocnemius muscles [13].

\section{Methods}

\section{Acquisition of Evidences}

In, this methodical review study a comprehensive online searching technique was applied for acquisition of evidences. The electronic databases: PubMed, Google scholar, Web of science, Embase, Medline, PsycINFO, ROAJ and DOAJR were sensibly searched to reviewing the literatures.

\section{Inclusion and Exclusion Criteria}

Studies related to the objective of this paper were included in this project whereas studies which were not directly matched with the concept of analysis were excluded from the procedure. Inclusion standards were incorporated with the areas of functional hamstring to quadriceps (H:Q) strength ratio, hamstring injury in soccer, Nordic hamstring exercise (NHE), eccentric strengthening of biceps femoris, lower extremity injury in soccer etc.

\section{Selection Procedure of Review Articles}

In the first attempt, a total 139 articles with full text were assessed on the basis of eligibility criteria. After literatures survey, out of which only 86 papers were, satisfied the objective of this study. Current researchers were tried to explore the allied area of $\mathrm{H}: \mathrm{Q}$ strength ratio and its mechanism for hamstrings injury but due to some limitations all the articles cannot discussed elaborately. Searches were limited to the entire 35 years which was 1984 to 2018. The final databases searches were conducted on August 2018. Articles were assessed on the basis of eligibility criteria first at the level of the title, abstract, source and then the full text article.

\section{Why Functional H:Q Ratio is Important for Sports Persons?}

There are several hamstrings and quadriceps muscular relationship that can be created from the different contraction modes. The main two muscular relationship are conventional $\mathrm{H}: \mathrm{Q}$ ratio and functional $\mathrm{H}: \mathrm{Q}$ ratio. The conventional $\mathrm{H}: \mathrm{Q}$ ratio is defined as the ratio between the peak torque of the hamstring and the quadriceps and is measured during a concentric contraction. While the functional $\mathrm{H}: \mathrm{Q}$ ratio is defined as the ratio between the peak torque of the hamstring during an eccentric contraction and the peak torque of the quadriceps during a concentric contraction $[9,14,15]$.

However, the conventional ratio uses a concentric contraction of both the hamstring and quadriceps muscle determining injury with value of $>0.6$ as abnormal [16]. Based on biomechanics of running or sprint the quadriceps contract concentrically to produce limb movement forward while at the same time the hamstring contracts eccentrically to slow down the limb movement, therefore, avoiding injury of the knee with control the whole-body motion [17]. Depending on the contraction adopted, muscle strength can be said to be isotonic, isokinetic or isometric [18]. Out of these three, isokinetic testing is reliable to measure functional H:Q ratio. Therefore, sports scientists used isokinetic testing to evaluate (H:Q) strength ratio [19-21]. Several sports professionals work on the $\mathrm{H}: \mathrm{Q}$ ratio and try to remove the inequity of this ratio. However, this ratio provides important information about the relative strength of different muscle groups, or muscle imbalances 9. Scientist postulate that greater than $15 \%$ difference between limbs is often considered a substantial asymmetry in healthy athletes and may put them at increased risk of injury [22]. There are two ways to recover $H: Q$ ratio. Player can either rise hamstrings strength or decrease quadriceps strength. Likewise, quadriceps or hamstrings strength alone does not relate to sports performance at all. The ratio of these two respective muscles is plays a very significant role. Most intermittent team sports need a lot of sprinting, running, jumping, acceleration, deceleration and sudden change of direction. Functional hamstring (eccentric): quadriceps (concentric) ratio is additional representative of fatigue induced by soccer than the conventional hamstring (concentric): quadriceps (concentric) ratio. Eccentric strength training at high velocities may be essential to decrease injury risk in soccer players [23]. Nevertheless, hamstrings and quadriceps muscles are antagonists to one another, simultaneous concentric contraction of both these muscles does not happen. Therefore, it may be preferred to examine eccentric hamstrings peak torque with respect to the concentric quadriceps peak torque when assessing functional strength [9]. A reduced H:Q ratio may be indicative of quadriceps dominance, may put athletes at a greater risk for lower extremity injury [22]. The functional ratio more realistically replicates the movement of an athlete and is reported to be normal at 1:1 (hamstrings: quadriceps) [24].

\section{Impact of H:Q Ratio with Team Games and Individual Sports}

Competitive sportspersons are required to perform explosive and powerful movements during many sporting events including 
soccer tournaments and track and field competitions. However, when evaluating strength, normative values provide a range of normal strength values for a population that can be used to compare collected data. Normative values are peak torque and $\mathrm{H}: \mathrm{Q}$ ratios at $60^{\circ} / \mathrm{s}$ and $300^{\circ} / \mathrm{s}$ [9]. Researchers found differences in the H:Q ratios among tennis, squash, and track athletes [25]. Differences in limb dominance with the conventional $\mathrm{H}: \mathrm{Q}$ ratio may not occur because specific loads imposed with training and competition may be similar for both limbs [26]. But, with respect to muscle strength in the dominant versus non-dominant leg, it has been proposed that there is an increased rate of injury when a difference of $15 \%$ or more in knee flexor or hip extensor strength occurs in collegiate athletes [22]. College athletes from a field sports may present with a lower H:Q ratio as a result of higher sprinting demands in the sport. In contrast, athletes from court sports may require stronger hamstrings to compensate more frequent alteration between lower extremity acceleration and deceleration due to a relatively smaller playing area [1]. The functional H:Q strength ratio is representative for the takeoff phase of a jump, where the quadriceps muscles contract and the shortening muscles extend the knee. In this situation eccentric (lengthening) contraction of the hamstring is required to slow down the explosive knee extension [9]. The anterior cruciate ligament (ACL), assisted by the hamstrings, stabilizes the knee by preventing the tibia from sliding out in front of the femur [27], which can occur during pivoting movements such as landing from a jump and sudden changes in direction in a field (soccer) and court (volleyball and basketball) athletes [28].

\section{Status of H:Q Ratio and Hamstrings Muscle Strength in Soccer}

Soccer injury can be occurred by two ways; a) contact injury, b) non-contact injury. There are several reasons for non-contact injury. According, to research report more than $50 \%$ of all injuries were to the lower extremity and most of them were noncontact injuries [29]. Researchers found that injured athletes had preseason $H: Q$ strength ratio of less than $0.6(\mathrm{p}<0.05)$. For the left leg injuries, the result revealed that statistically significant relationship found between the lower preseason HQ ratio $(<0.6)$, and the number of the lower leg injuries [30]. The hamstrings muscle injury occurred while it stretched beyond its capacity to its standard stretched. The hamstrings group is reported to be the most common injury representing more than one third of all muscle stretched where $70 \%$ have an acute outset and in non-contact situation in $90 \%$ [31]. Scientist examine that, hamstring(ecc): quadriceps(con) of semi-professional soccer players before and after a soccer-specific field test and found a significant $16.8 \%$ decrease in functional hamstring(ecc): quadriceps(con) ratio [32]. A healthy hamstring to quadriceps muscle strength range of $50-80 \%$ is widely accepted [33]. Alternatively, H:Q of $<60 \%$ increases the risk of injury [30]. Researchers found that soccer specific fatigue test i.e. Loughborough Intermittent Shuttle Test (LIST), led to a decreased capacity of the hamstrings to counteract the force of the quadriceps, as reflected by a lower hamstring (ecc):quadriceps(con) ratio and suggest that eccentric strength training at high velocities might be useful to prevent injuries in soccer players [23].

Functional hamstring(ecc): quadriceps(con) strength ratio seems to better reproduce the knee extension and flexion pattern used for instance in soccer kicking. In this regard eccentric hamstrings assessment follows the co-activation pattern developed in leg deceleration phase during the final angles of knee extension $[14,15]$. Moreover, investigators, found a lower H:Q strength ratio was a significant predictor for traumatic injuries of the lower extremity in general [34]. Professional soccer participation presents a high risk of hamstring muscle injury, both during competition and training [35]. Adolescent soccer players had markedly higher physical fitness compared with untrained adolescents [36]. Winning the football match depend on several factors and physical fitness is one of them. The athletes who did not regularly exercise their hamstrings had a significant decrease in hamstrings activation in compare with normal healthy subjects [26].

\section{Impact of Hamstrings Muscle Injury on Soccer Players}

Over, the last few years' researchers found a gradual increase in the proportion of hamstrings strains compare to other injury types and it is the number one lesion in football [31]. Hamstring's strain is among the most common injury in sports that involve sprinting, turning, and jumping [37]. Acute hamstring strains often result in significant recovery time and have a lengthy period of increased susceptibility for recurrent injury [38].

Muscles of the hamstring are prone to be affected due to imbalances of H:Q strength ratio. Most of the hamstring's injury occurred in biceps femoris-84\%, semimembranosus-11\%, semitendinosus-5\% [31]. However, a prospective analysis of all academy-aged players from an English Premier League club during one season and assessed 93 players out of which 20 players suffered hamstring injuries that season [39]. Researchers reported that subcategorized hamstrings risk factors divided into two main sections, i.e. nonmodifiable factors which are issues that cannot be changed, whereas the modifiable factors can be changed $[40,41]$. As per previous research earlier injury, age and dark or aboriginal ethnic origin are the most common nonmodifiable factors [37,4244]. Aboriginal players are considered to be the fastest and most skillful players; they are more likely to have a greater proportion of type II (fast twitch) muscle fibers that in turn may predispose them to injury [45]. However, it has been postulated that type II muscle fibers are more susceptible to injury because of the faster contraction times or stretch being placed upon a muscle when it is contracting [46]. Scientists also focused on the most prominent and researched factors which are modifiable: low hamstring strength, fatigue, quadriceps flexibility, tiredness and fitness [41]. In fact, at the pre-swing position of sprinting, the rectus femoris muscle is 
lengthened and acts eccentrically to arrest extension of the hip and flexion of the knee. In this stretched position, its tendon absorbs energy to be released during the active flexion of the hip and knee, when the leg is accelerated forward. If the rectus femoris muscle is tight; however, there may be a rise in the passive elastic recoil of the tendon, further increasing the acceleration of the leg. This increases the load on the hamstrings, thereby increasing the risk of injury [41]. Hamstring to quadriceps concentric strength ratio (H:Q) of the injured leg was approximately lower than that of the uninjured leg. According to previous examines, it has been argued that $\mathrm{H}: \mathrm{Q}$ concentric strength is not a functional assessment and thus, not related to the mechanisms of injury. A more functional assessment of strength is to express the $\mathrm{H}: \mathrm{Q}$ ratio for terms of hamstring eccentric strength to quadriceps concentric strength [41]. With this in mind, the eccentric torque produced by the hamstrings would need to match the concentric torque of the quadriceps to prevent possible injury, that is, a functional hamstring(ecc): quadriceps(con) strength ratio of 1:1 (hamstrings: quadriceps). This has been supported by previous research $[47,48]$.

\section{Importance of Biceps Femoris Long Head (Bflh) Muscle for Injury Prevention}

The biceps femoris (long head) inserted into the medial portion of the upper half of the ischial tuberosity via a thick, round tendon. The proximal tendon of biceps femoris employed the lateral one quarter of the upper medial portion of the ischial tuberosity and this proximal tendon of biceps femoris long head was comparatively long, with a mean length of $27.1 \mathrm{~cm}$, extending to occupy $61.9 \%$ of the length of the muscle [49]. Biceps femoris long head (BFlh) is the most frequently injured part of whole hamstrings muscles (84\%) [31]. This muscle enables to lengthening at highest level while semimembranosus generates the greatest amount of force with most power. So, it is necessary to need different methods of injury prevention technique for specific muscle. Biceps femoris need lengthen training, and the semimembranosus necessity strengthening approach. As the most majority of hamstrings injury are located at the biceps femoris. So, most treatments should take on this muscle which is work by lengthening under load [31]. Similarly, the effect of eccentric isokinetic loading on the quadriceps and hamstrings musculature is relatively unexplored. During eccentric contraction muscle exerts a force by developing tension and controlling its lengthening in the direction of the opposing force [50]. The presence of short biceps femoris long head (BFlh) fascicles and low levels of eccentric knee flexor strength in elite soccer players increases the risk of future hamstring strain injury (HSI). The greater risk of a future HSI in older players or those with a previous HSI is reduced when they have longer BFlh fascicles and high levels of eccentric strength [51].

\section{Eccentric Strength Reduce the Hamstrings Injury}

Eccentric muscle contractions produce high forces with lowenergy cost [52] are now well-established properties of eccentric contractions. Developing eccentric muscle contraction can reduce the rate of injury. This injury occurs when a muscle is eccentrically overstretched, typically during the late swing phase of sprinting [53]. Many researchers suggest that if the hamstrings are not eccentrically strong enough then its lengthening capacity would be less and tight. Tight Hamstrings also can limit the forward motion of pelvis and bring the pelvis back slightly along with straighten the lower spine. The primary role of the hamstrings in walking and running is to eccentrically control the landing of the foot. Additionally, the hamstring complex undergoes a substantial eccentric contraction during the late swing phase [54]. Scientists are trying to test the relationship between eccentric training and subsequent injury in elite male soccer players and found eccentric training group significantly fewer hamstring injuries compared to the control group [55]. In addition, the training group showed statistically significant improvements in strength and speed [55]. Other researchers investigated the effect of eccentric hamstring strengthening post soccer training and observed significantly reduced the effects of fatigue. Therefore, performing eccentric training with fatigue may have a time-dependent beneficial effect [56]. Since soccer involves many movement's and skills for covering a big field, players should always be ready for transition from attack to defense as well as defense to attack. To link up with these transitions players always accelerate, decelerate; sometimes change their direction according to necessity on match. To perform these motor movements soccer players, need a sufficient amount of eccentric strength of hamstring.

\section{Nordic Hamstring Exercise and other Treatments for Developing Eccentric Strength}

The Nordic hamstring exercise (NHE) has been shown to be an effective tool for developing higher maximal eccentric hamstring strength torques when compared with regular hamstrings curl [57]. Since, Nordic hamstring curl exercise can be a good tool to maintain $\mathrm{H}: \mathrm{Q}$ muscular strength ratio. Investigators adopting the NHE in regular training on male professional soccer players and found reduced hamstring injury incidence rates by $65 \%$ to $70 \%$, with a particularly preventive effect in reducing recurrent injuries [58,59]. Furthermore, NHE would build eccentric strength, muscular hypertrophy, running speed, acceleration and definitely reduces the risk of hamstrings injury. The Nordic hamstring exercise could decrease of $71 \%$ of hamstring lesions and reduction of $86 \%$ of re-injury [31]. However, $\mathrm{H}: \mathrm{Q}$ strength ratio can vary according to sporting background [24], training, gender [33]. The H:Q ratio are significantly different in dominant and non-dominant legs. Several researchers proposed Nordic hamstring strengthening exercise may reduce the rate and severity of hamstring muscle injuries [41,57-59]. Scientist Mjølsnes and his team suggested the complete nordic hamstring curl exercise schedule (Figure 1) [57]. According to authors the participants are instructed to let themselves fall forward and then resist the fall against the gravity as long as possible by using their hamstrings while their feet are kept on the 
ground by a partner (Figure 2) [57]. The Nordic hamstring curl can be done by two ways: assisted and unassisted method [41]. Scientist also recommended that the load is increased as participant can withstand the forward fall longer. When managing to withstand the whole range of motion (ROM) for [12] repetition, increase load by adding speeds to the starting phase of the motion. The partner can also increase loading further by pushing at the back of shoulders [57]. However, Nordic hamstring curl exercise should be done by systematic, minimal, and progressive way. Increase in volume, reps, and load of this exercise must maintain.

\begin{tabular}{|cccc|}
\hline Week & Sessions perweek & Sets & Repetitions \\
\hline 1 & 1 & 2 & 5 \\
2 & 2 & 2 & 6 \\
3 & 3 & 3 & $6-8$ \\
4 & 3 & 3 & $8-10$ \\
$5-10$ & 3 & 3 & $12,10,8$ \\
\hline
\end{tabular}

Figure 1: Complete Nordic hamstring curl exercise schedule (Mjolsnes, Arnason, osthagen, Raastad, \& Bahr, 2004).

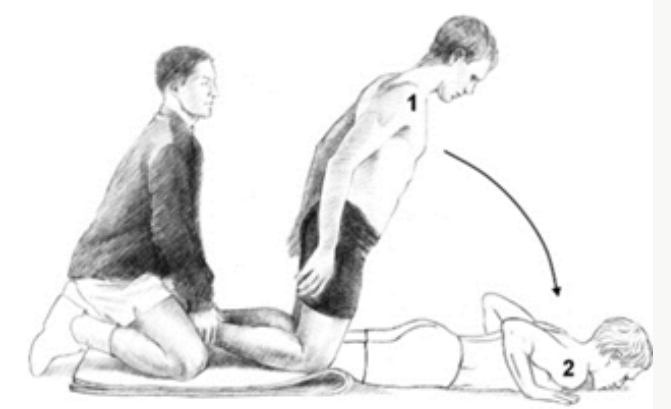

Figure 2: Customary Nordic hamstring exercise (NHE) (Mjolsnes et al., 2004).

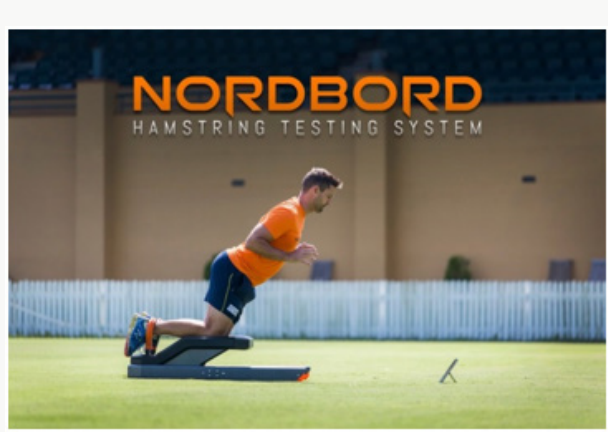

Figure 3: Nordic hamstring exercise on scientific nord board (Developed by Vald performance).

Alternatively, a scientific Nord board is a fast and easy way to accurately measure eccentric and isometric hamstring strength (Figure 3). The English Premier League title winner (2015-16 seasons) Leicester City club applied the scientific nord board for built player's hamstring strength eccentrically. Nevertheless, there are a lot of other exercises to develop and maintain hamstrings strength as well as hamstrings to quadriceps strength ratio. These are FIFA 11+ injury prevention programme, total resistance exercise (TRX) suspension training, single leg deadlift, hamstrings curl on swiss ball, bridge on foam roller, etc. The FIFA 11+ warm-up program $30 \%$ reduced the risk of injury in soccer players [40].

\section{Conclusion}

Hamstring to quadriceps strength ratio need to be maintaining regularly to settle all discrepancy. The ratio of hamstring to quadriceps may be a predictor of hamstrings strain [31]. Nordic hamstring curl (assisted and unassisted method) can increase the eccentric strength of hamstrings and minimize the risk of injury. All training program should try to prevent as many sport injuries as possible and help athletes to improve performance [60]. Therefore, it can be noted that there is clearer relationship between injury and performance. On, the contrary the coach and trainer is not always responsible for maintaining every player's $\mathrm{H}: \mathrm{Q}$ ratio. Trend of sports science at present is clearer so time has come that player should aware their $\mathrm{H}: \mathrm{Q}$ strength ratio matter. To clarify, only player can feel the pain and recognize the internal condition of hamstrings.

\section{Limitations}

The current study focusses on cause of hamstrings injury with $H: Q$ ratio but there are other existing reasons for hamstrings injury such as age, fatigue, muscle strength, flexibility, previous injury, core stability, ethnicity, tiredness, fitness, lumbosacral nerve impingement etc. are not discussed.

\section{Acknowledgement}

The researchers are thankful to Indian Council for Cultural Relations (ICCR), Ministry of External Affairs and University Grant Commission (UGC), Ministry of Human Resource Development, Govt. of India for their financial consideration.

\section{Highlights}

a) Hamstring to quadriceps strength ratio prevents hamstring injury.

b) Nordic hamstring curl exercise can increase the eccentric strength of hamstrings and minimize the risk of injury.

c) Every player needs to maintain hamstring to quadriceps strength ratio for higher sports performance.

\section{References}

1. Cheung R, Smith A, Wong D (2012) H:Q Ratios and Bilateral Leg Strength in College Field and Court Sports Players. J Hum Kinet 33: 63-71.

2. Magalhães J, Oliveira J, Ascensão A, Soares J (2004) Concentric quadriceps and hamstrings isokinetic strength in volleyball and soccer players. J Sports Med Phys Fitness 44(2): 119-125. 
3. Calmels P, Minaire P (1995) A review of the role of the agonist/antagonist muscle pairs ratio in rehabilitation. Disabil Rehabil 17(6): 265-276.

4. Hewett TE, Myer GD, Zazulak BT (2008) Hamstrings to quadriceps peak torque ratios diverge between sexes with increasing isokinetic angular velocity. J Sci Med Sport 11(5): 452-459.

5. Liu H, Garrett WE, Moorman CT, Yu B (2012) Injury rate, mechanism, and risk factors of hamstring strain injuries in sports: A review of the literature. J Sport Health Sci 1(2): 92-101.

6. Heiser TM, Weber J, Sullivan G, Clare P, Jacobs RR (1984) Prophylaxis and management of hamstring muscle injuries in intercollegiate football players. Am J Sports Med 12(5): 368-370.

7. Orchard J (2002) Epidemiology of injuries in the Australian Football League, seasons 1997-2000. Br J Sports Med 36(1): 39-44.

8. Upton PA, Noakes TD, Juritz JM (1996) Thermal pants may reduce the risk of recurrent hamstring injuries in rugby players. Br J Sports Med 30(1): 57-60.

9. Willigenburg NW, McNally MP, Hewett TE (2018) Quadriceps and Hamstrings Strength in Athletes. In: Kaeding CC, Borchers JR, editors. Hamstring and Quadriceps Injuries in Athletes. Boston, MA: Springer US, p.15-28.

10. Carlson C (2008) The natural history and management of hamstring injuries. Curr Rev Musculoskelet Med 1(2): 120-123.

11. Heiderscheit BC, Sherry MA, Silder A, Chumanov ES, Thelen DG (2010) Hamstring Strain Injuries: Recommendations for Diagnosis, Rehabilitation, and Injury Prevention. J Orthop Sports Phys Ther 40(2): 67-81.

12. Corazza A, Orlandi D, Baldari A, Gatto P, Stellatelli M, et al. (2014) Thigh muscles injuries in professional soccer players: A one-year longitudinal study. Muscles Ligaments Tendons J 3(4): 331-336.

13. Valle X, L Tol J, Hamilton B, Rodas G, Malliaras P, et al. (2015) Hamstring Muscle Injuries, a Rehabilitation Protocol Purpose. Asian J Sports Med 6(4): e25411.

14. Aagaard P, Simonsen EB, Trolle M, Bangsbo J, Klausen K (1995) Isokinetic hamstring/quadriceps strength ratio: Influence from joint angular velocity, gravity correction and contraction mode. Acta Physiol Scand 154(4): 421-427.

15. Aagaard P, Simonsen EB, Magnusson SP, Larsson B, Dyhre-Poulsen P (1998) A New Concept for Isokinetic Hamstring: Quadriceps Muscle Strength Ratio. Am J Sports Med 26(2): 231-237.

16. Wright J, Ball N, Wood N (2009) Fatigue, H/Q ratios and muscle coactivation in recreational football players. Isokinet Exerc Sci 17(3): 161-167.

17. Croisier JL (2004) Muscular imbalance and acute lower extremity muscle injuries in sport. Int Sport Med J 5(3): 169-176.

18. Jaiyesimi AO, Jegede JS, Adeoluwa OJ (2007) Hamstring and Quadriceps Strength Ratio: Effect of Age and Gender. Highl Med Res J 15(2): 5.

19. Li RC, Maffulli N, Hsu YC, Chan KM (1996) Isokinetic strength of the quadriceps and hamstrings and functional ability of anterior cruciate deficient knees in recreational athletes. Br J Sports Med 30(2): 161-164.

20. Pinto MD, Blazevich AJ, Andersen LL, Mil-Homens P, Pinto RS (2018) Hamstring-to-quadriceps fatigue ratio offers new and different muscle function information than the conventional non-fatigued ratio. Scand J Med Sci Sports 28(1): 282-293.

21. Sabino GS, Felício DC, Guimarães CQ, Abreu BJ da GA, Vieira WH de B (2016) Validity analysis of one-repetition maximum strength test for determining the hamstrings-to-quadriceps ratio. Mot Rev Educ Física 22(3): 133-137.

22. Knapik JJ, Bauman CL, Jones BH, Harris JM, Vaughan L (1991) Preseason strength and flexibility imbalances associated with athletic injuries in female collegiate athletes. Am J Sports Med 19(1): 76-81.
23. Delextrat A, Gregory J, Cohen D (2010) The Use of the Functional H:Q Ratio to Assess Fatigue in Soccer. Int J Sports Med 31(3): 192-197.

24. Holcomb WR, Rubley MD, Lee HJ, Guadagnoli MA (2007) Effect of hamstring-emphasized resistance training on hamstring: Quadriceps strength ratios. J Strength Cond Res 21: 41-47.

25. Read MT, Bellamy MJ (1990) Comparison of hamstring/quadriceps isokinetic strength ratios and power in tennis, squash and track athletes. Br J Sports Med 24(3): 178-182.

26. Rosene JM, Fogarty TD, Mahaffey BL (2001) Isokinetic Hamstrings: Quadriceps Ratios in Intercollegiate Athletes. J Athl Train 36(4): 378383.

27. Pettitt RW, Bryson ER (2002) Training for Women's Basketball: A Biomechanical Emphasis for Preventing Anterior Cruciate Ligament Injury. Strength Cond J 24(5): 20.

28. Griffin LY, Agel J, Albohm MJ, Arendt EA, Dick RW, et al. (2000) Noncontact Anterior Cruciate Ligament Injuries: Risk Factors and Prevention Strategies. J Am Acad Orthop Surg 8(3): 141-150.

29. Hootman JM, Dick R, Agel J (2007) Epidemiology of collegiate injuries for 15 sports: summary and recommendations for injury prevention initiatives. J Athl Train 42(2): 311-319.

30. Kim D, Hong J (2011) Hamstring to Quadriceps Strength ratio and Noncontact Leg Injuries: A Prospective Study During One Season: 957. Med Sci Sports Exerc 43: 138.

31. Michel Hooghe (2016) The number one lesion in football. In: Chall Footb Inj Conf Aspetar-Isakos-FIFA, Qatar.

32. Small K, McNaughton L, Greig M, Lovell R (2010) The effects of multidirectional soccer-specific fatigue on markers of hamstring injury risk. J Sci Med Sport 13(1): 120-125.

33. Kong PW, Burns SF (2010) Bilateral difference in hamstrings to quadriceps ratio in healthy males and females. Phys Ther Sport 11(1): 12-17.

34. Söderman K, Alfredson H, Pietilä T, Werner S (2001) Risk factors for leg injuries in female soccer players: A prospective investigation during one out-door season. Knee Surg Sports Traumatol Arthrosc 9(5): 313-321.

35. Dauty M, Menu P, Fouasson-Chailloux A (2018) Cutoffs of isokinetic strength ratio and hamstring strain prediction in professional soccer players. Scand J Med Sci Sports 28(1): 276-281.

36. Hammami A, Randers MB, Kasmi S, Razgallah M, Tabka Z, et al. (2018) Effects of soccer training on health-related physical fitness measures in male adolescents. J Sport Health Sci 7(2): 169-175.

37. Bennell K, Wajswelner H, Lew P, Schall-Riaucour A, Leslie S, et al. (1998) Isokinetic strength testing does not predict hamstring injury in Australian Rules footballers. Br J Sports Med 32(4): 309-314.

38. Sherry MA, Best TM (2004) A Comparison of 2 Rehabilitation Programs in the Treatment of Acute Hamstring Strains. J Orthop Sports Phys Ther 34(3): 116-125.

39. Rolls A, George K (2004) The relationship between hamstring muscle injuries and hamstring muscle length in young elite footballers. Phys Ther Sport 5(4): 179-187.

40. Sadigursky D, Braid JA, De Lira DNL, Machado BAB, Carneiro RJF, et al.(2017) The FIFA 11+ injury prevention program for soccer players: A systematic review. BMC Sports Sci Med Rehabil 9: 18.

41. Turner AN, Cree J, Comfort P, Jones L, Chavda S, et al. (2014) Hamstring Strain Prevention in Elite Soccer Players. Strength Cond J 36(5): 10-20.

42. Gabbe BJ (2005) Risk factors for hamstring injuries in community level Australian football. Br J Sports Med 39(2): 106-110.

43. Verrall GM, Slavotinek JP, Barnes PG, Fon GT (2003) Diagnostic and Prognostic Value of Clinical Findings in 83 Athletes with Posterior Thigh Injury: Comparison of Clinical Findings with Magnetic Resonance 
Imaging Documentation of Hamstring Muscle Strain. Am J Sports Med 31(6): 969-973.

44. Woods C (2004) The Football Association Medical Research Programme: An audit of injuries in professional football--analysis of hamstring injuries. Br J Sports Med 38(1): 36-41.

45. Verrall GM (2001) Clinical risk factors for hamstring muscle strain injury: A prospective study with correlation of injury by magnetic resonance imaging. Br J Sports Med 35(6): 435-439.

46. Blankenbaker DG, De Smet AA (2004) MR imaging of muscle injuries. Appl Radiol 33: 14-26.

47. Croisier J-L, Forthomme B, Namurois M-H, Vanderthommen $M$ Crielaard J-M (2002) Hamstring Muscle Strain Recurrence and Strength Performance Disorders. Am J Sports Med 30(2): 199-203.

48. Yamamoto T (1993) Relationship between hamstring strains and leg muscle strength. A follow-up study of collegiate track and field athletes. J Sports Med Phys Fitness 33(2): 194-199.

49. Woodley SJ, Mercer SR (2005) Hamstring Muscles: Architecture and Innervation. Cells Tissues Organs 179(3): 125-141.

50. Dean E (1988) Physiology and Therapeutic Implications of Negative Work A Review. Phys Ther 68(2): 233-237.

51. Timmins RG, Bourne MN, Shield AJ, Williams MD, Lorenzen C, et al. (2016) Short biceps femoris fascicles and eccentric knee flexor weakness increase the risk of hamstring injury in elite football (soccer): A prospective cohort study. Br J Sports Med 50(24): 1524-1535.

52. Ortega JO, Lindstedt SL, Nelson FE, Jubrias SA, Kushmerick MJ, et al. (2015) Muscle force, work and cost: A novel technique to revisit the Fenn effect. J Exp Biol 218(13): 2075-2082.
53. Chumanov ES, Schache AG, Heiderscheit BC, Thelen DG (2012) Hamstrings are most susceptible to injury during the late swing phase of sprinting. Br J Sports Med 46(2): 90.

54. Yu B, Queen RM, Abbey AN, Liu Y, Moorman CT, et al. (2008) Hamstring muscle kinematics and activation during overground sprinting. J Biomech 41(15): 3121-3126.

55. Askling C, Karlsson J, Thorstensson A (2005) Hamstring injury occurrence in elite soccer players after preseason strength training with eccentric overload. Scand J Med Sci Sports 13(4): 244-250.

56. Small K, McNaughton L, Greig M, Lovell R (2009) Effect of Timing of Eccentric Hamstring Strengthening Exercises During Soccer Training: Implications for Muscle Fatigability. J Strength Cond Res 23(4): 10771083.

57. Mjolsnes R, Arnason A, osthagen T, Raastad T, Bahr R (2004) A 10-week randomized trial comparing eccentric vs. concentric hamstring strength training in well-trained soccer players. Scand J Med Sci Sports 14(5): 311-317.

58. Arnason A, Andersen TE, Holme I, Engebretsen L, Bahr R (2007) Prevention of hamstring strains in elite soccer: an intervention study: Prevention of hamstring strains in soccer. Scand J Med Sci Sports 18(1): 40-48.

59. Petersen J, Thorborg K, Nielsen MB, Budtz-Jørgensen E, Hölmich P (2011) Preventive Effect of Eccentric Training on Acute Hamstring Injuries in Men's Soccer: A Cluster-Randomized Controlled Trial. Am J Sports Med 39(11): 2296-2303.

60. Rahnama N (2012) Preventing Sport Injuries: Improving Performance. Int J Prev Med 3(3): 143-144.

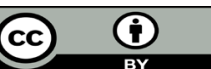

This work is licensed under Creative Commons Attribution 4.0 License

To Submit Your Article Click Here:

Submit Article

DOI: $10.32474 /$ OSMOAJ.2018.02.000133

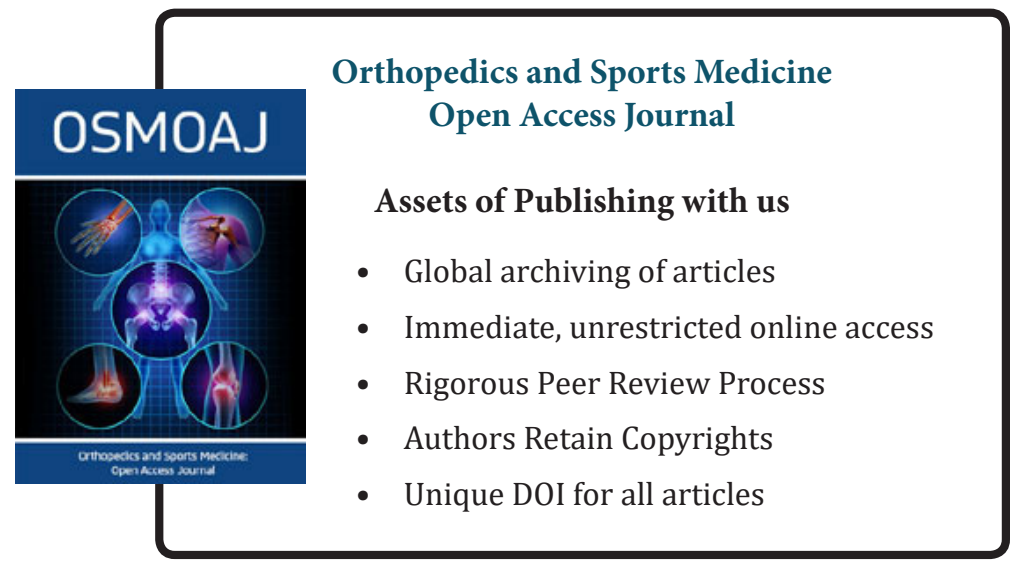

